\title{
Discerning the Potential of Chitosan Extracted from Bio resources as a Competent Brain Drug Carrier to Cross the Blood Brain Barrier
}

\author{
A. ANITA MARGRET*, T. NARGIS BEGUM ${ }^{1}$ AND A. GANESH KUMAR ${ }^{2}$ \\ Department of Biotechnology and Bioinformatics, Bishop Heber College, ${ }^{1}$ Department of Biotechnology, Jamal \\ Mohamedh College, 'Laboratory of DNA Bar coding and Marine Genomics, Department of Marine Science, \\ Bharathidasan University, Tiruchirappalli-620 024, India
}

Anita, et al.: Discerning the Proficiency of Chitosan as a Competent Brain Drug Carrier

\begin{abstract}
Biopolymer such as chitosan is more favoured as a drug carrier in particular to the brain due to its potential to cross the blood brain barrier. This study is a comparative assessment of the extraction of chitosan from natural sources such as mushroom, crab and shrimp using alkali method pursued by physicochemical assays that surmise the excellence of the biopolymer in permeating a central nervous system drug. Commercially, chitosan extracted from crustaceans have been widely used but this study revealed that mushrooms are also a good source for chitosan. An in silico assay enumerates the structural affiliation of the polymer and its binding efficiency. Molecular docking study facilitates to attest the brain targeting efficiency of chitosan on disparity with sodium alginate against the efflux permeability glycoprotein furnishing minimum binding affinity energy values of $\mathbf{- 3 7 . 2} \mathrm{k} \mathrm{cal} / \mathrm{mol}$. The pharmacologically significant descriptors propose a low solubility that avoids the untargeted discharge of drug. Chitosan extracted from a rich protein source such as mushroom in a mild alkaline condition with a high degree of deacetylation has freer amine groups can act as a drug by itself and can readily bind with a drug as a carrier. The synchronised work of biopolymer extraction with that of in silico analysis can further take chitosan as a lead compound for brain drug development and targeting studies.
\end{abstract}

Key words: Biopolymer, chitosan, central nervous system, molecular docking, blood brain barrier, permeability, glycoprotein

Polymers partake a vital role in drug delivery system as they control the release of the drug from a delivery system. Natural polymers promote drug delivery due to their physicochemical characteristics, ease of availability, biodegradablenature and lack of appreciable toxicity. Hence, biopolymers exhibit the potential to enhance medical treatment by minimizing adverse effects. Biopolymers are polymers produced by living organisms and chitosan is a natural polymer obtained by deacetylation of chitin. Further it has better stability and versatility of being applicable to various routes of drug administration ${ }^{[1]}$. The deacetylated chitosan consists of glucosamine units as the backbone, which has a high density of charged amine groups, permitting strong electrostatic interactions with proteins and genes that carry an overall negative charge at neutral $\mathrm{pH}^{[2]}$. The physicochemical properties of chitosan play a significant role in drug delivery that has been illustrated quite elaborately in the literature ${ }^{[3]}$. Commercial chitosan is extracted from crustaceans, but is subjected to harsh chemical treatments. Chitosan is produced from shrimp and crab shell chitin by deacetylation using strong alkalis at high temperatures for long periods of time ${ }^{[4]}$. However, supplies of raw materials have been variable with season due to the laborious and costly process. Furthermore, chitosan derived from such a process is heterogeneous with respect to physiochemical properties ${ }^{[5]}$. Considering these aspects there is a necessity to perform a comparative study of the extraction and characterization of chitosan from all

This is an open access article distributed under the terms of the Creative Commons Attribution-NonCommercial-ShareAlike 3.0 License, which allows others to remix, tweak, and build upon the work non-commercially, as long as the author is credited and the new creations are licensed under the identical terms

Accepted 18 February 2017

Revised 19 October 2016

Received 15 October 2015

Indian J Pharm Sci 2017;79(2):186-196 
three bio resources such as crab, shrimp and mushroom using a mild alkaline method. Moreover, chitosan extracted from mushroom was found to be on par with that extracted from crustaceans and could be used for various medical applications ${ }^{[6]}$. On the large scale, high amounts of chitosan is extracted from crustaceans, but advances in fermentation technology render the cultivation of selected mushrooms an alternative source of chitosan ${ }^{[7]}$.

Polymers exhibit a good potential for surface modification by chemical transformations, which increases the solubility index ${ }^{[8]}$. This remarkable potential of biodegradable polymers is to provide greater pharmacokinetic control, which makes them suitable for entrapment and delivery of a wide range of therapeutic agents ${ }^{[9]}$. Chitosan has the potential of serving as an absorption enhancer across epithelial membranes due to its mucoadhesive and absorption enhancement properties. This distinguished feature of chitosan is likely to enhance the drug dispersion in the gastrointestinal tract that ultimately improves bioavailability of various drugs. Drugs targeting central nervous system (CNS) must cross the blood brain barrier (BBB), which is the main obstacle for a variety of molecules to enter the CNS tissue ${ }^{[10]}$. The use of biocompatible nanoparticles to facilitate the traverse of drugs across BBB has been extensively researched in the past decade ${ }^{[11]}$. In drug delivery, selection of an ideal type of biopolymer like chitosan might result in sustaining drug delivery to prolong the duration of action, improve therapeutic efficiency and simultaneously reduce side effects. Chitosan might as well aid in brain drug targeting by being an ideal carrier for drugs through the BBB. The present study aimed at developing an alternate bio resource to produce chitosan with a comparative physicochemical profile. The present investigation also employed docking analysis to investigate the brain targeting capability of the biopolymer.

\section{MATERIALS AND METHODS}

All chemicals used were of the highest available commercial grade. Commercial chitosan from shrimp shells ( $\geq 85 \%$ deacetylated) was purchased from HiMedia Laboratories (Mumbai, India) and other chemicals such as sodium hydroxide, hydrochloric acid, ethylenediaminetetraacetic acid (EDTA) and bleaching powder were obtained from Scientific Research Laboratory Ltd (Chennai, India).

\section{Collection of samples:}

Commercially available freshly packed edible mushrooms were purchased from super market store. The mushroom species was authenticated as Agaricus bisporus (No. AA 001) in the Rapinat Herbarium and Centre for Molecular Systematic, St. Joseph's College Tiruchirappalli, Tamil Nadu, India. The exoskeleton of crab and prawn was collected from the central market of Tiruchirappalli, Tamil Nadu, India.

\section{Extraction of chitosan:}

The purchased mushroom (A. bisporus) samples were washed exhaustively with water after adjusting $\mathrm{pH}$ with $\mathrm{KOH}$ to 8-9. Chitosan was extracted from the washed mushrooms with $\mathrm{KOH}$ and $\mathrm{HCl}$ using a method developed by combination and modification of protocols implied earlier ${ }^{[12,13]}$. Mushroom biomass was treated with $60 \%(\mathrm{w} / \mathrm{v})$ aqueous $\mathrm{KOH}$ solution $(10 \mathrm{ml}$ per $1 \mathrm{~g}$ of dry mycelium) at $130^{\circ}$ for $2-3 \mathrm{~h}$ to remove proteins, lipids and alkali-soluble polysaccharides. For chitosan isolation, this freeze-dried alkali insoluble fraction was boiled with $0.1-1 \%(\mathrm{v} / \mathrm{v})$ acetic acid solutions (50 ml per $1 \mathrm{~g}$ of dry matter) for 10-40 min. The $\mathrm{pH}$ value of the acidic supernatant was increased to 9.5 with $2 \mathrm{M} \mathrm{NaOH}$. The alkali-insoluble precipitate (presumable chitosan) obtained was repeatedly centrifuged and washed with distilled water, freeze dried and weighed.

The shells of crab and prawn were washed, dried and powdered. The powdered exoskeletons were suspended in boiling $\mathrm{NaOH}$ ( 2 and $4 \% \mathrm{w} / \mathrm{v}$ ) for 1 $\mathrm{h}$, which facilitated the isolation of crude chitosan by dissolving the proteins and sugars. The samples were treated in two different concentrations of $\mathrm{NaOH}$ where, the higher concentration (4\%) is used for chitin preparation $^{[14,15]}$. Further at room temperature the shells were subjected to demineralization using $1 \%$ $\mathrm{HCl}$ where, the minerals (mainly calcium carbonate) are removed ${ }^{[16]}$. The demineralization process was preceded by treating the samples with $50 \mathrm{ml}$ of a $2 \%$ $\mathrm{NaOH}$ solution to decompose the albumen into water soluble amino acids. The remaining chitin was washed and further converted into chitosan by the process of deacetylation ${ }^{[17]}$, which was carried out by boiling the samples in $50 \% \mathrm{NaOH}$ for $2 \mathrm{~h}$ on a hot plate. Repeated washing with $50 \% \mathrm{NaOH}$ and filtration retained a solid matter, thus extracting chitosan as whitish flakes. The obtained chitosan was purified to increase its commercial value using three process such as filtration, reprecipitation and demineralisation ${ }^{[18]}$. The insoluble 
fraction of the sample was removed by filtration through Whatman filter paper $22 \mu \mathrm{m}$. Chitosan was precipitated from filtered chitosan solution by titration with $1 \mathrm{~N} \mathrm{NaOH}$ until a $\mathrm{pH}$ value of 8.5 is attained. The chitosan so obtained was washed several times with distilled water by centrifuging at 8000 to $10000 \times \mathrm{g}$. Demineralisation is the final process in the extraction of chitosan where, $1 \mathrm{ml}$ of $10 \% \mathrm{w} / \mathrm{v}$ aqueous sodium dodecyl sulphate (SDS) was used for dissolving the remaining protein. The samples treated with SDS overnight were exposed with $3.3 \mathrm{ml}$ of $5 \% \mathrm{w} / \mathrm{v}$ EDTA for complexing heavy metals with EDTA. Repeated centrifugation extracts the insoluble fractions and the residue obtained is dried and used for commercial purpose.

\section{Characterization of prepared chitosan:}

A homogeneous mixture of $1 \mathrm{mg} / \mathrm{ml}(\mathrm{w} / \mathrm{v})$ concentration of chitosan solution was prepared with $1 \%$ glacial acetic acid to analyse and characterise various parameters such as degree of deacetylation (DD), $\mathrm{pH}$, ash value, loss on drying and assaying its functional group. DD referred to removal of acetyl group from the chain, which was determined by potentiometric titration $^{[19]}$. DD $\%=100-\mathrm{DA}$. The $\mathrm{pH}$ measurements of the chitosan solutions were carried out using a $\mathrm{pH}$ a.

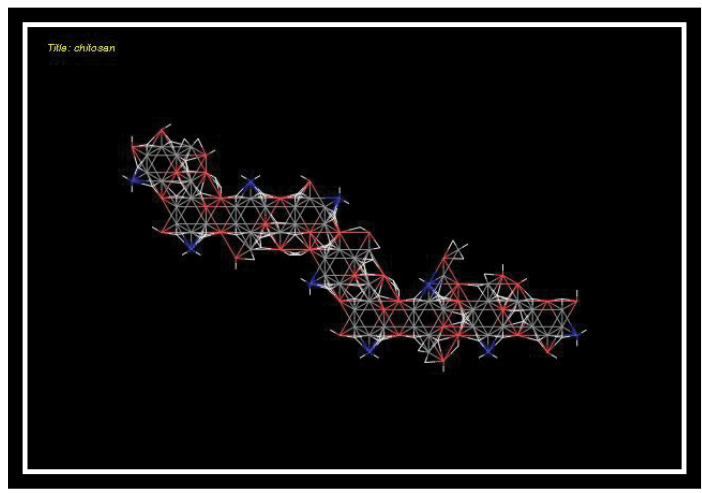

c.

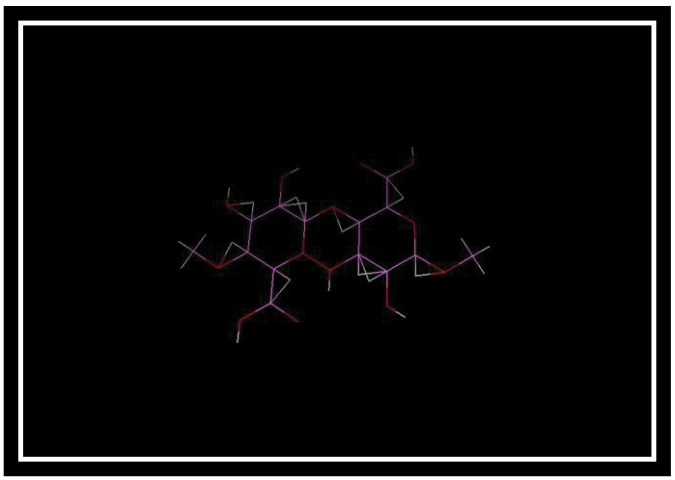

meter (Elico model no IL120). To determine the ash value of chitosan, samples were heated in a muffle furnace and the percentage of ash value was calculated using the following Eqn. ${ }^{[20]}$, ash value (\%) = weight of the residues $(\mathrm{g}) /$ weight of the sample $(\mathrm{g}) \times 100$. Loss on drying of the prepared chitosan was determined by the gravimetric method of Black ${ }^{[21]}$, the result of which was expressed as \% loss on drying, which was (wet weight-dry weight)/dry weight $\times 100$.

Fourier transform infrared spectroscopy (FTIR) spectroscopy of solid samples of the extracted chitosan was done on a Bio-Rad FTIS-40 Model USA. Ten grams of sample was mixed with $100 \mathrm{~g}$ of dried potassium bromide $(\mathrm{KBr})$ and compressed to prepare a salt disc (10 $\mathrm{mm}$ diameter) for reading the spectrum further. The FTIR profile of the standard chitosan was analysed using a FTIR spectrum.

\section{Preparation of chitosan:}

The original ligand structures of the target was drawn and prepared (fig. 1a-c). The structures of the chitosan (CID: 71853), sodium alginate (CID: 6850754) and chitosan oligosaccharide lactate (CID: 16213812) were downloaded from Pubchem. The $\mathrm{H}$ bonds were fixed and the energy minimized structures were generated using ACD/ChemSketch ACD (2009).

b.

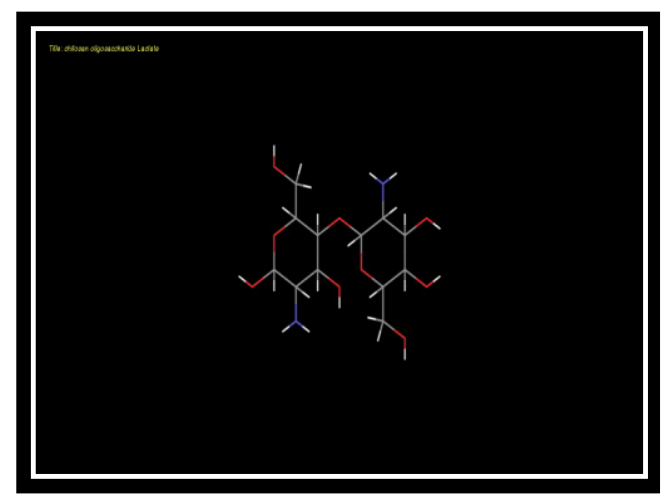

d.

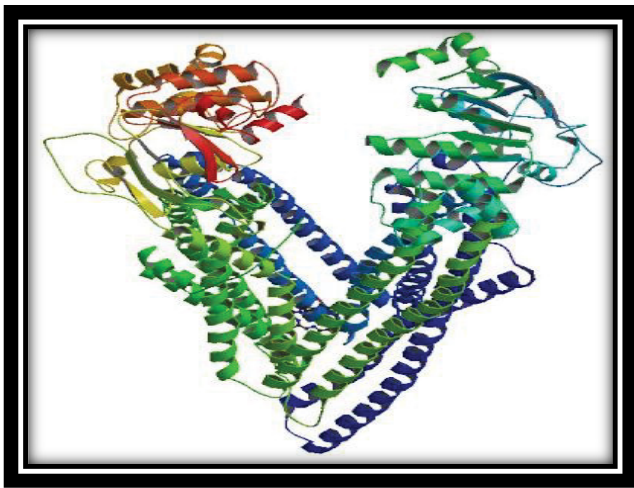

Fig. 1: Structures of the biopolymers as ligands and target protein a. Chitosan (CID No: 71853); b. sodium alginate (CID No: 6850754); c. chitosan oligosaccharide lactate (CID No: 16213812); d. crystal structure of P-gp (PDB No: 4M1M) 
Docking of the chitosan and hits with the target:

The ligand compounds folder was loaded. Population size was set to 200. Number of generations was set to 25 and 2 best poses were retrieved. From the output folder, the binding energy for the best pose of the ligand was noted. The hydrogen bond interaction between the ligand and the binding site residues were analysed and interaction table was created and saved. The best compounds were identified based on the energy values and the fitness score was calculated based on the total energy of a predicted pose in the binding site.

\section{Absorption, distribution, metabolism, and excretion-toxicity (ADMET) property prediction and molecular docking studies:}

Molecular docking evaluates the interaction of chitosan

a.

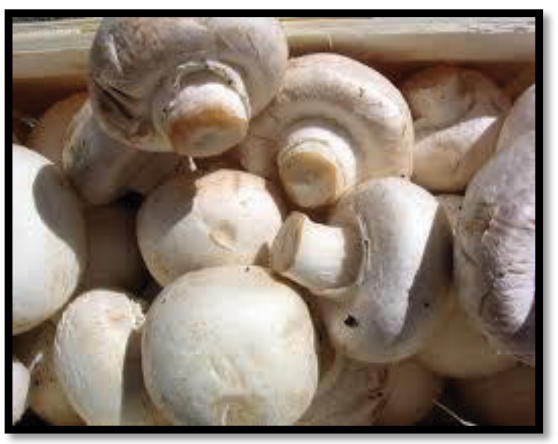

b.

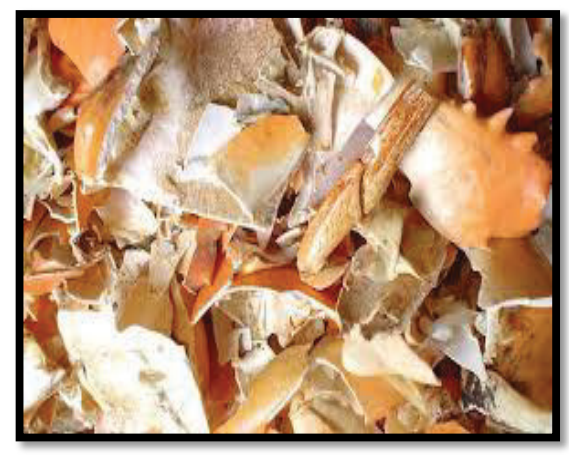

c.

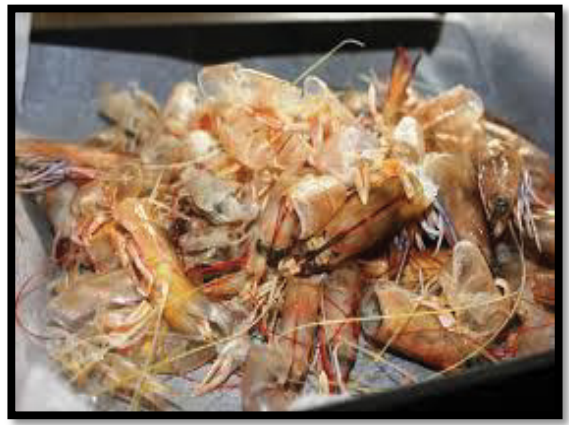

and the targeted protein permeability glycoprotein (P-gp) using Hex 8.0.0 software. The study also incorporated the drug delivery related properties of chitosan by structural activity relationship using Medchem Designer version 3.0. The target protein, P-gp was downloaded from Protein Data Bank (PDB ID $4 \mathrm{M} 1 \mathrm{M}$, fig. 1d). The structures were visualized using the molecular graphics program PyMol intended for the structural visualization of proteins, nucleic acids and small biomolecules and then loaded into Hex. The binding site was specified exactly based on the ligand group. The distance of the binding site was set to $8 \AA$.

\section{RESULTS AND DISCUSSION}

Crude chitosan was extracted from all three bio resources, mushroom, crab and prawn (fig. 2a, b
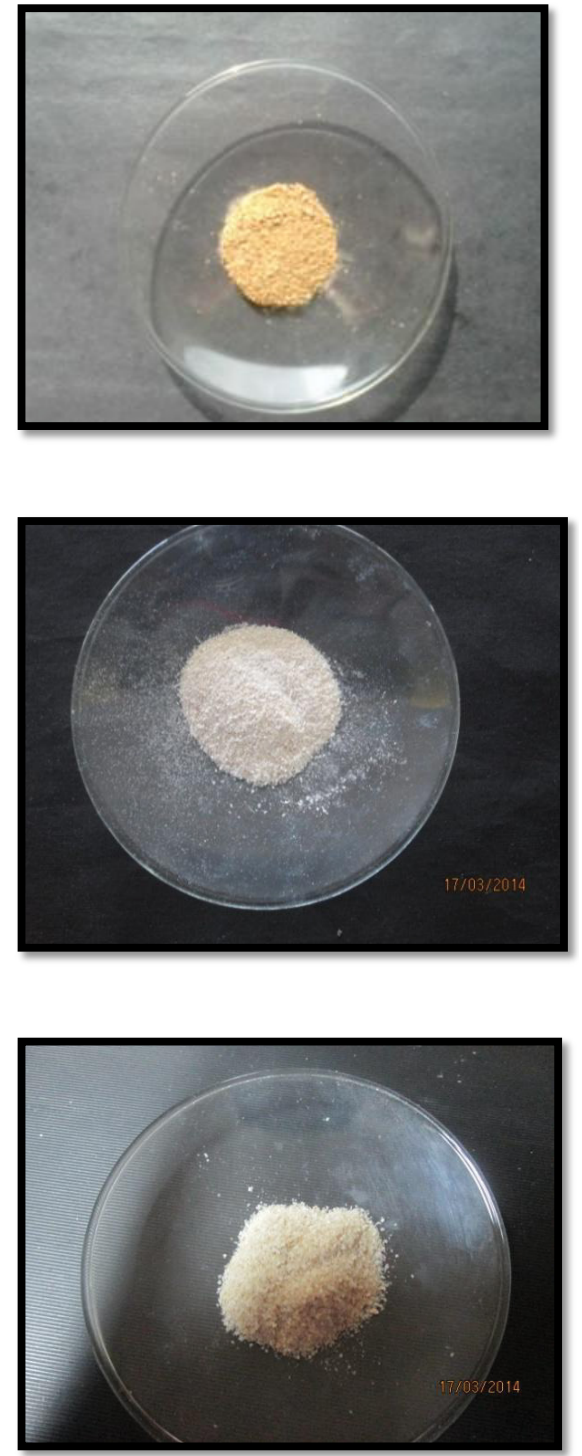

Fig. 2: Chitosan extracted from various bio sources

Bio source and the appearance of chitosan extracted from those. Chitosan from a. mushroom; b. crab; c. prawn 
and c) with the removal of proteins followed by demineralization, which included the eradication of carbon and other salts proceeded by the deacetylation of the chitin. The extracted product was creamy white in colour with a crumble texture. When subjected to light the sharp edged flakes glittered. The percent biomass, in the production of crude chitosan was found to be, crab $5.8 \mathrm{~g}$, (17.46\%), prawn $5.2 \mathrm{~g}$, (15.65\%) and mushroom $4.6 \mathrm{~g}(13.85 \%)$. The $\mathrm{pH}$ analysis of the extracted sample ensured alkalinity at 8.5.

The loss of water content from chitosan extracted from mushroom, crab, prawn and standard (commercial sample) was evaluated. All the three bio resources elected as test samples are moist with a constant and equivalent moisture content. But, the extracted chitosan from these test samples exhibited distinctive values that differ from each other. Eventually the moisture value of commercial chitosan is $<10 \%{ }^{[22]}$ and fig. 3a represented a bar graph illustration, where the moisture value of crab chitosan is in close proximity to the standard chitosan. Polymers like chitosan were semi crystalline (partially crystalline amorphous) in nature hence it would be difficult to interpret the structure if the moisture content is high. Crystals are commonly nano-scaled in size and self-assembled nanostructures were proved to be highly effective in drug delivery ${ }^{[23]}$. Hence, chitosan should be dry and as per the data received compared to the standard, the extracted biopolymer from crustaceans has a high percent loss on drying as compared to that from mushroom. This may be due to their habitat and influence of salinity. The ash content from the extracted chitosan of all three bio resources was compared with that of the standard. As evaluated with the commercial sample meagre differences were found in the chitosan extracted from crab and mushroom. The ash values are analogous to each other (fig. 3b), which could be interpreted as due mainly to the inorganic content present in the biopolymer. Minerals are elements that aid in structure formation partaking as components of biologically active molecules with beneficial effects ${ }^{[24]}$. The luminal membrane of the brain endothelium (facing blood capillary) is lined with ATP binding cassette family of active efflux transporter pumps like P-gp, multidrug resistance protein (MRP) and on the abluminal surface of choroid plexus is the multi specific organic anion transporter (MOAT) ${ }^{[25,26]}$. They act as a restraint to remove the un-intended drugs/substances (lipophilic/ neurotoxic) from the endothelia cell cytoplasm, before it could cross the brain parenchyma ${ }^{[27]}$ hence, there is a need of a carrier, which can pass through this efflux protein. Chitosan has an adhesion property that plays an important role in the interaction of its positively charged amino group with the negatively charged sites on the epithelial surface. It has been reported that chitosan can adhere to the mucosal surface and transiently open the tight junctions between epithelial cells, thus it's an effective absorption enhancer. There is a need of energy-dependent process and divalent cations such as $\mathrm{Ca}^{2+}$ to regulate the tight junction permeability ${ }^{[28]}$. Therefore the presence of inorganic contents and minerals benefits chitosan as a carrier into the $\mathrm{BBB}$ and mushroom expedites as a bio resource with a characteristic ash value.

The DD of the extracted chitosan from crab, prawn and mushroom was assayed by means of potentiometric titration along with the standard chitosan. DD is one of the main parameters characterizing chitosan. The calculated values are depicted with a graphical representation (fig. 3c). The extracted chitosan from all three resources comprised of DD value of $\geq 75$, which confirmed that chitin is deacetylated and it becomes soluble in the acid. The process of deacetylation involves the removal of acetyl groups from the molecular chain of chitin, leaving behind the biopolymer (chitosan) with a high degree chemically reactive amino groups $\left(-\mathrm{NH}_{2}\right)$. This makes the DD an important property in chitosan production as it has a significant role in endorsing physicochemical characterisation thereby enhancing drug delivery ${ }^{[29]}$. A polymer with a high deacetylation value supplements the required protein constitution that reduces immunogenicity and limits the phagocytosis of drug by the reticuloendothelial system. This results in an increased drug concentration in blood and vital organs such as the brain, intestines and kidneys ${ }^{[30]}$.

Chitosan is a cationic polymer that has the capacity to chemically bind with negatively charged macromolecules. Hence, chitosan have attained increasing commercial interest as suitable resource materials due to its biocompatibility, biodegradability, adsorption properties that facilitate into epithelial cells. The crystallinity of the chitosan increases with the increase in DD and is attributed to increase in intermolecular hydrogen bonding due to the presence of more free $\mathrm{NH}_{2}$ groups (higher DD) within the molecular structure, which in turn results the better packing of the macromolecular polymeric chains and consequent increase in the crystallinity ${ }^{[31]}$. Crystals in nanoscale has the enhancement of penetration into 
a.

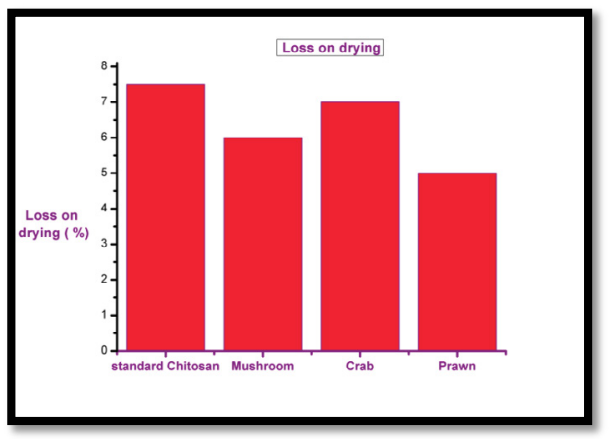

b.

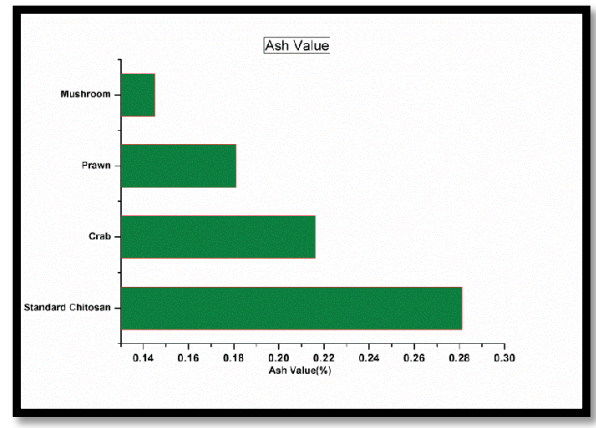

c.

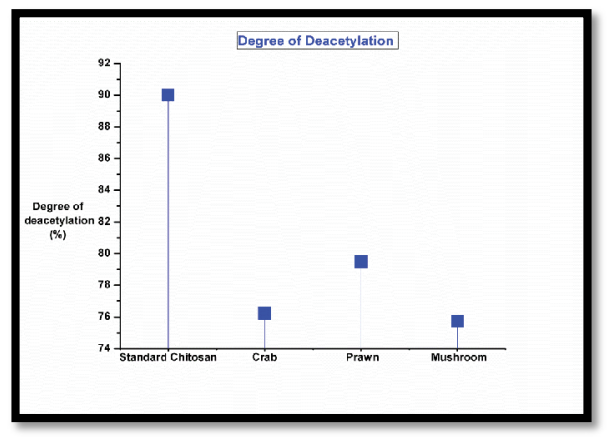

Fig. 3: Comparative analyses of extracted chitosan with commercial sample a. Loss on drying; $b$. ash value; c. degree of deacetylation

biological membranes, hence a polymer like chitosan with a good DD can act as an incredible brain drug carrier to penetrate into the BBB.

The FTIR study allowed the comparison of the functional groups in all the three samples extracted from different bio sources with the standard. Fig. 4a, b, $\mathrm{c}$ and d illustrated the spectral details of the extracted chitosan from three bio resources, crab (S1), prawn (S2) and mushroom (S3) besides the standard chitosan (S) and Table 1 listed the FTIR peak assignments of all the four samples. In order to characterize the test samples, spectrum of standard chitosan (S) is recorded with distinctive peaks having major absorption band at $3435 \mathrm{~cm}^{-1}$ due to stretching vibrations of $\mathrm{OH}$ groups. Relatively the chitosan sample extracted from S2 and S3 had the same groups at $3465 \mathrm{~cm}^{-1}$ and $3423 \mathrm{~cm}^{-1}$. Whereas the spectral profile of $\mathrm{S} 1$ had a discrete band at $3233 \mathrm{~cm}^{-1}$ owing to the $\mathrm{NH}_{2}$ stretching vibration. Asymmetric stretching vibrations of $\mathrm{C}-\mathrm{H}$ bond in $\mathrm{CH}_{2}$ and $-\mathrm{CH}_{3}$ attributed to the pyranose ring at 2922 $\mathrm{cm}^{-1}, 2862 \mathrm{~cm}^{-1}$ featured in the spectrum of standard chitosan ( $\mathrm{S}$ ), where the $\mathrm{C}-\mathrm{H}$ bond in $-\mathrm{CH}_{2}$ pyranose ring is identical in all the three samples $\mathrm{S} 1\left(2913 \mathrm{~cm}^{-}\right.$ $\left.{ }^{1}\right), \mathrm{S} 2\left(2931 \mathrm{~cm}^{-1}\right)$ and S3 $\left(2926 \mathrm{~cm}^{-1}\right)$. Absorption at the range of $1649 \mathrm{~cm}^{-1}, \mathrm{~S}$ corresponds to the vibrations of carbonyl bonds $-\mathrm{C}=\mathrm{O}$ in acetamide group (amide $\mathrm{I}$ band). This functional groups closely relates itself to the biopolymer and similar spectral peaks were found in S2 $\left(1612 \mathrm{~cm}^{-1}\right)$ and S3 $\left(1647 \mathrm{~cm}^{-1}\right)$. The bending vibrations of spectral peaks of S1 $\left(1595 \mathrm{~cm}^{-1}\right)$ and S3 $\left(1570 \mathrm{~cm}^{-1}\right)$ are associates with the protonated amine group. Bending vibrations of methylene and methyl groups were evident at 1379 and $1427 \mathrm{~cm}^{-1}$ in the standard sample ${ }^{[32]}$. Respectively, all the three samples have the presence of bending vibrations $\delta(\mathrm{C}-\mathrm{H})$ at $\mathrm{S} 1$ $\left(1421 \mathrm{~cm}^{-1}\right), \mathrm{S} 2\left(1415 \mathrm{~cm}^{-1}\right), \mathrm{S} 3\left(1417 \mathrm{~cm}^{-1}\right)$ and 1380 and $1321 \mathrm{~cm}^{-1}$ at $\mathrm{S} 1$ and $\mathrm{S} 2$. Absorption in the range from 1160 to $1000 \mathrm{~cm}^{-1}$ has been attributed to vibrations of CO group ${ }^{[33]}$. The band located at $1153 \mathrm{~cm}^{-1}$ in $\mathrm{S}$ and $\mathrm{S} 1$ is related to asymmetric vibrations of in the oxygen bridge resulting from deacetylation of chitosan. The bands near to $1095-1025 \mathrm{~cm}^{-1}$ are attributed to the stretching vibrations of $\mathrm{C}-\mathrm{O}$ in the ring of $\mathrm{COH}, \mathrm{COC}$ and $\mathrm{CH}_{2} \mathrm{OH}$, which is uniformly found in all three test samples and standard. The extracted chitosan was authenticated with the standard biopolymer using FTIR studies. All the three extracted samples have similar functional groups there by characterizing itself as chitosan.

Molecular docking and ADMET predictions are two imperative in silico techniques used to unveil the pharmaceutical significance of chitosan. The binding efficiency of the biopolymer was evaluated against the efflux protein P-gp and tabulated in Table 2. The 
a.

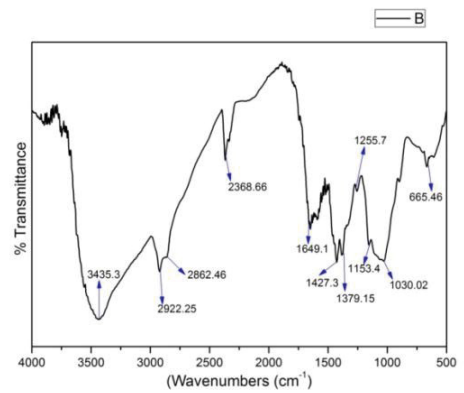

c.

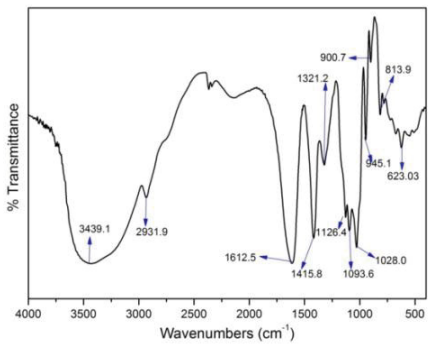

b.

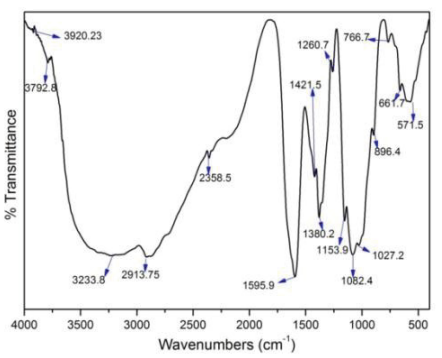

d.

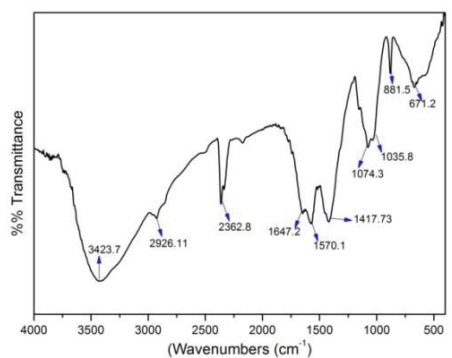

Fig. 4: The FTIR spectra of chitosan

a. Standard-S; b. crab-S1; c. prawn-S2; d. mushroom-S3

TABLE 1: WAVELENGTH OF THE MAIN BANDS OBTAINED FOR THE STANDARD CHITOSAN AND CHITOSAN EXTRACTED FROM MUSHROOM, CRAB AND PRAWN

\begin{tabular}{lcccc}
\hline Vibration mode* & Standard chitosan & Crab & Prawn & Mushroom \\
\hline$v$ s( C-O) & 1030 & 1027 & 1028 & 1035 \\
& - & 1082 & 1093 & 1074 \\
$v$ as(C-O-C) & 1153 & 1153 & 1126 & - \\
$v(\mathrm{C}-\mathrm{O})$ & 1255 & 1260 & - & - \\
$\delta(\mathrm{C}-\mathrm{H})$ & 1379 & 1380 & 1321 & - \\
& 1427 & 1421 & 1415 & 1417 \\
$(-\mathrm{C}=\mathrm{O})$ (amide I band) & 1649 & - & 1612 & 1647 \\
$\delta(\mathrm{NH})$ & - & 1595 & - & 1570 \\
$v$ as(C-H) & 2922 & 2913 & 2931 & 2926 \\
$v(\mathrm{NH})$ & - & 3233 & - & - \\
$v(\mathrm{O}-\mathrm{H})$ & 3435 & - & 3439 & 3423 \\
\hline
\end{tabular}

${ }^{*} \mathrm{v}=$ stretching vibration; $\mathrm{v} \mathrm{s}=$ symmetric stretching vibration; $\mathrm{v}$ as = asymmetric stretching vibration; $\delta=$ bending vibration

TABLE 2: BINDING AFFINITY VALUE BY MOLECULAR DOCKING AGAINST P-GP

\begin{tabular}{lc}
\hline Ligand & Affinity (kcal/mol) \\
\hline Chitosan & -27.4 \\
Chitosan oligosaccharide lactate & -23.18 \\
Sodium alginate & -37.2 \\
\hline
\end{tabular}

binding site of the target was prepared and the energy minimized compounds were imported. Docking studies are devised for virtual screening to analyse the potential of the compound acting as a drug. This works deals with the study of analysing the efficiency of chitosan as a potential drug as a carrier transient through the BBB. The permeability glycoprotein or plasma glycoprotein is an active, efflux, membrane bound transport protein pump ${ }^{[34]}$. Though chitosan has established itself as an efficient drug carrier, its high molecular weight intervene the interaction between the proteins. Hence, chitosan derivative such as chitosan oligosaccaharide lactate is incorporated as drug delivery system while encapsulating ocular drugs ${ }^{[35]}$.

Based on the experimental study, chitosan is docked against $\mathrm{P}$-gp through in silico rigid docking approach where the ligand is flexible and protein is static. The docking results showed that both the ligands (chitosan and its derivative) interacted with the target protein (P-gp surrounded by several donor atoms of chitosan 
residue) on the surface with a free binding energy of -27.4 and $-23.18 \mathrm{kcal} / \mathrm{mol}$ (fig. 5a and b) whereas, sodium alginate (reference polymer) interacted with a minimum affinity value of $-37.2 \mathrm{kcal} / \mathrm{mol}$. Though the minimum energy score of chitosan oligosaccharide lactate was greater than sodium alginate, it exhibited interaction with the protein through the residues PHE 974, PHE 724 (fig. 5c and d). Interestingly, these residues also responded with the bioactive co crystallized ligand flavin-adenine dinucleotide (FAD) and antidepressant drug, venlafaxine ${ }^{[36]}$. The docking simulation studies of chitosan deduce PHE 974, PHE 724 as interacting key residues, which also found in the standard antidepressant drug venlafaxine and ligand FAD. The molecular modelling showed the participation of biopolymer with the protein by means of $\mathrm{H}-\mathrm{OH}$ interaction. Hence, the biopolymer has the prospective of inhibiting the efflux protein P-gp, thereby facilitating the drug through the BBB.

An idyllic drug carrier releases the drug precisely under the varying environmental stimuli. Computational studies like structure activity relationship (SAR) aids in designing a smart delivery system, which is prudent and efficient. In concern to this, a preliminary study has undergone highlighting the pharmacologically significant descriptors. Some calculated structure activity values $(\log \mathrm{P}, \log \mathrm{D}$, topological polar surface area and Hydrogen bond donor and acceptor

a.

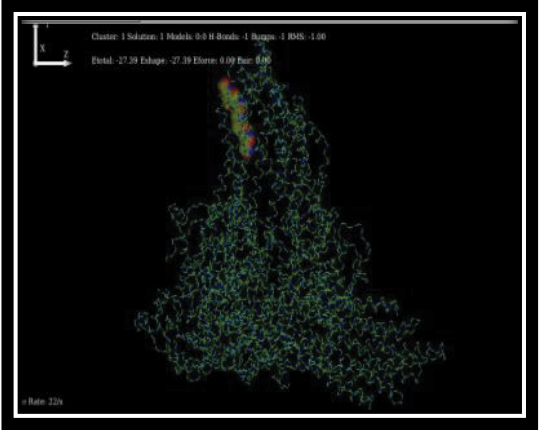

c.

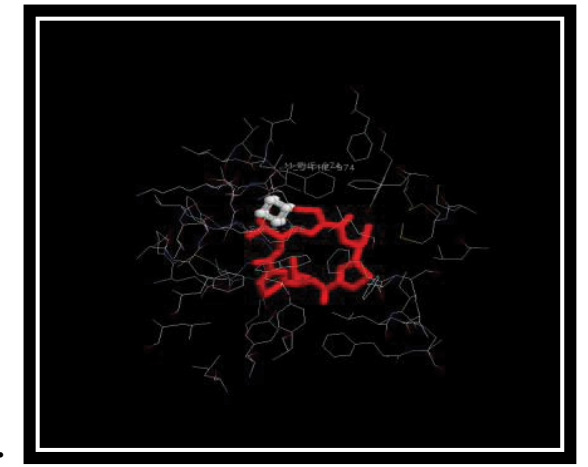

protons) of the biopolymers correlates to the ADMET properties of a drug. Table 3 represented the values of all three-test polymers. Aqueous solubility is one prime pharmacological property of a drug carrier and some have low solubility values ${ }^{[37]}$. A drug carrier with a high solubility index has the chance to disintegrate thereby dispersing the drug before it could reach the target, which influences the bioavailability of the encapsulated $\operatorname{drug}^{[38]}$.

Hence, drug carriers with low solubility value are constant leading in the definite distribution of drugs to the targeted sites. Partition-coefficient (P) or distribution-coefficient (D) is the proportion of a drug in a mixture of two immiscible phases, which is the indicator of solubility. Log $\mathrm{P}$ value is the measure of lipophilicity of a compound that assesses its dispersion in a medium ${ }^{[39]}$. The physiochemical descriptors illustrate a low $\log P$ value for chitosan $(-12.000)$ when compared to chitosan oligosaccharide lactate $(-3.264)$ and sodium alginate $(-5.208)$. Hence, the dispersion rate of chitosan is more in aqueous medium and prevents the disintegration of the drug from reaching the target. The dissociation factor of a drug carrier influences the distribution coefficient value of drug even at varied physiological condition. Deviation in $\mathrm{pH}$ is due to the persistence of abnormal physiological condition and a drug carrier should be able to resist and continue stable. Log D value (Table 3 ) indicates

b.

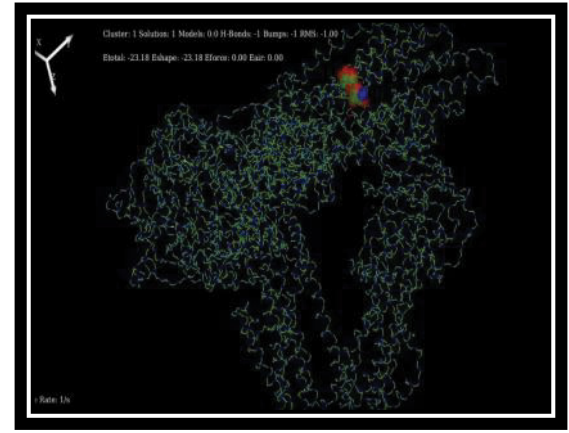

d.

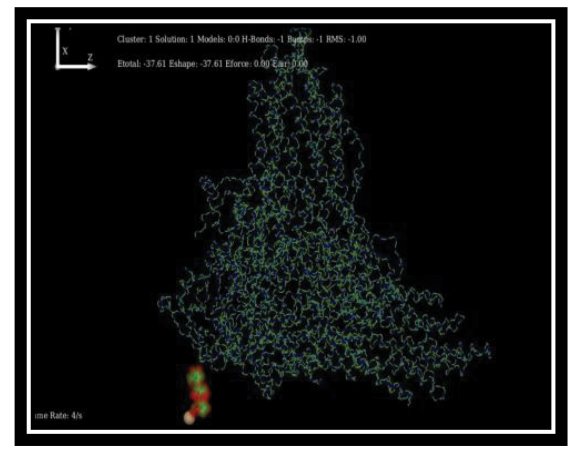

Fig. 5: Best docked conformations of drug polymers

a. Chitosan with the protein P-gp; b. chitosan oligosaccharide lactate with the protein P-gp; c. interaction of the ligand chitosan oligosaccharide lactate (red) within the active site residues of the target; $d$. sodium alginate with the protein P-gp 
the dissociation at the specified $\mathrm{pH} 7.4$ where, chitosan dissociates low $(-13.945)$ and prolongs to be so in the acidic conditions. It then gradually increases in the basic condition and this was similar in the $\log \mathrm{D}$ values of chitosan oligosaccharide lactate $(-4.283)$, which eventually increased in the basic conditions. But sodium alginate did not confer any disparity in the $\log \mathrm{D}$ value between the acidic and basic conditions and among the acidic state -5.028 persist to be the unvarying.

Lipinski's rule attests the ability of a drug by devising rules that an ideal drug should not violate all the four rules. As mentioned earlier in the study high molecular weight of chitosan dissuades the drug likeness parameter whereas, chitosan oligosaccharide regulates this by persuading 3 rules of Lipinski (Table 4). Hence, the study elucidates chitosan as a drug carrier with more biocompatibility surpassing the BBB.

The present study evidently unveils the significance of chitosan acting as suitable brain drug carrier. All though the extraction of this biopolymer from natural resources like crab, prawn and mushroom has been pioneered and customized commercially, this study attempts to axis the brain drug delivery to a distinct facet. On a large scale, chitosan is preferred to be extracted from crustaceans but, edible mushrooms like $A$. bisporus also has the potential to confer and this has been attested with an appreciable physicochemical characterization. The extraction of chitosan from mushroom was at ease, which also gave satisfactory results on par with the other bio resources and the method of extraction is malleable, when compared to the harsh chemical treatments. High DD and ash value infers the quality and drug binding mechanism of the polymer. These characterizations were performed to associate with the brain drug facilitation across the BBB. The docking stimulus interactions of chitosan and the targeted protein P-gp further substantiate the plausibility in facilitating brain drug across the epithelial barrier. As drug carriers the biopolymers should possess the capability of controlled drug release and the structural activity relationship studies paves way to determine the significant descriptors. The comparison of the predicted properties such as $\log \mathrm{P}$ and $\log \mathrm{D}$ were comparatively less. A low solubility in concert with a customary $\mathrm{pH}$ averts the adverse dispersal of drugs to the untargeted sites. Being a natural polymer chitosan and its derivatives are advantageous over other drug carriers. Application of nano science can assist the biopolymer coated drugs in reducing and avoiding adverse effects, thereby minimizing the duration of treatment. Neurological diseases are a threat to standard living and drug loaded with the extracted chitosan as would be an excellent remedy and can improve the quality of therapeutic value.

\section{Acknowledgments:}

The authors acknowledge the support offered by the management of Bishop Heber and Jamal Mohamedh College, Tiruchirappalli, Tamil Nadu, India. Facilities provided by the Laboratory of DNA Bar coding and Marine Genomics, Department of Marine Science Bharathidasan University, Tiruchirappalli, Tamil Nadu, India are gratefully acknowledged. The authors also thank Dr. S. John Britto, Director, Rapinat Herbarium and Centre for Molecular Systematic, St. Joseph's College Tiruchirappalli, for his assistance in authenticating the mushroom species.

\section{Conflict of interest:}

The authors declare no conflicts of interest.

\section{Financial support and sponsorship:}

Nil.

TABLE 3: SOME OF THE CALCULATED ADMET DESCRIPTORS AS DRUG CARRIERS

\begin{tabular}{lccccc}
\hline Drug carriers & log P & $\log \mathrm{D}$ & $\begin{array}{c}\text { Topological polar } \\
\text { surface area }\end{array}$ & $\begin{array}{c}\text { HBDH No of } \\
\mathrm{H}_{2} \text { bond donor } \\
\text { protons }\end{array}$ & $\begin{array}{c}\mathrm{M} \mathbf{N O}_{-} \mathrm{No}_{2} \text { of } \mathrm{N}_{2} \text { and } \\
\mathrm{O}_{2} \text { atoms }\end{array}$ \\
\hline Chitosan & -12.000 & -13.945 & 5808.00 & 37.00 & 48.00 \\
$\begin{array}{l}\text { Chitosan oligosaccharide } \\
\text { lactate }\end{array}$ & -3.264 & -4.00 & 6201.11 & 11.00 & 10.0 \\
Sodium alginate & -5.208 & -5.208 & 320.14 & 16.00 & 19.00 \\
\hline
\end{tabular}

TABLE 4: CALCULATED PROPERTIES OF LIPINSKI'S RULES FOR THE DRUG DELIVERY SYSTEMS

\begin{tabular}{|c|c|c|c|c|}
\hline Drug carriers weight donor & Molecular bond acceptor & Hydrogen bond & Hydrogen & $\log P$ \\
\hline Chitosan & 1526.49 & 37.00 & 48.00 & -12.000 \\
\hline Chitosan Oligosaccharide Lactate & 340.332 & 11.00 & 10.00 & -3.264 \\
\hline Sodium Alginate & 554.459 & 16.00 & 19.00 & -5.208 \\
\hline
\end{tabular}




\section{REFERENCES}

1. Pacheco N, Gamica-Gonzalez M, Gimeno M, Barzana E, Trombotto S. Structural characterization of chitin and chitosan obtained by biological and chemical methods. Biomacromolecules 2011;12:3285-90.

2. Rabea EI, Badawy MET, Stevens CV, Smagghe G, Steurbaut W. Chitosan as antimicrobial agent: application and mode of action. Biomacromolecules 2003;4:1457-65.

3. Sanyakamdhorn S, Agudelo D, Tajmir-Riahi HA. Encapsulation of antitumor drug doxorubicin and its analogue by chitosan nanoparticles. Biomacromolecules 2013;14:55763.

4. Knorr D. Recovery and utilization of chitin and chitosan in food processing waste management. Food Technol 1991;26:114-22.

5. Crestini C, Kovac B, Giovannozzi-Sermanni G. Production and isolation of chitosan by submerged and solid-state fermentation from Lentinus edodes. Biotechnol Bioeng 1996;50:207-10.

6. Khor E, Lim LY. Implantable applications of chitin and chitosan. Biomaterials 2003;24:2339-2349.

7. Ruiz-Herrera J, Sentandreu RR, Martinez JP. Chitin biosynthesis in fungi. In: Dilip KA, Richard PE, Mukerji $\mathrm{KG}$, editors. Handbook of Applied Mycology, Fungal Biotechnology. 4th ed. New York: Marcel Dekker; 1992. p. 281-312.

8. Alyautdin RN, Reichel A, Lobenberg R, Ramge P, Kreuter $\mathrm{J}$, Begley DJ. Interaction of poly (butylcyanoacrylate) nanoparticles with the blood brain barrier in vivo and in vitro. J Drug Target 2001;9:209-21.

9. Kreuter J, Ramge P, Petrov VE, Hamm S, Gelperina SE, Engelhardt B, et al. Nanoparticle drug delivery system and genes carrier drug delivery. J Pharm Res 2003;20:409-16.

10. Blasi P, Giovagnoli S, Schoubben A, Ricci M, Rossi C. Solid lipid nanoparticles for targeted brain drug delivery. Adv Drug Deliv Rev 2007;59:454-77.

11. Wang J, Valmikinathan $\mathrm{CM}, \mathrm{Yu} \mathrm{X}$. Nanostructures for bypassing blood brain barrier. Curr Bioact Compd 2009;5:195205.

12. Muzzarelli RAA, Rochetti R. Determination of the degree of deacetylation of chitosan by first derivative ultraviolet spectrophotometry. J Carbohydr Polym 1985;5:461-72.

13. White SA, Farina PR, Fulton I. Production and Isolation of Chitosan from Mucor rouxii. Appl Environ Microbiol 1979;38:323-8.

14. Lertsutthiwong P, How NC, Chandrkrachan S, Stevens WF. Effect of chemical treatment on the characteristics of shrimp chitosan. J Min Met Mat S 2002;12:11-18.

15. Lamarque GJ, Lucas M, Viton C, Domard A. Physicochemical behaviour of homogeneous series of acetylated chitosan in aqueous solution: role of various structural parameters. Biomacromolecules 2005;6:131-42.

16. Trung TS, Thein-Han WW, Qui NT, Stevens WF. Functional characteristics of shrimp chitosan and its membranes as affected by the degree of deacetylation. Bioresour Technol 2006;97:659-63.

17. Huang M, Khor E, Lim LY. Pharmaceutical Research, Uptake and cytotoxicity of chitosan molecules and nanoparticles: Effects of molecular weight and degree of deacetylation. Pharm Res 2004;21:344-53.

18. Qian RQ, Glanville RW. Methods for purifying chitosan.
Providence Health System. US Patent Number-6896809; 2005.

19. Zhanga $Y$, Zhanga X, Dinga R, Zhanga J, Liub J. Determination of the degree of deacetylation of chitosan by potentiometric titration proceeded by enzymatic pre-treatment. Carbohydr Polym 2011;83:813-7.

20. MonarulIslama Shah MD, Masumb M, Mahbubur Rahma-na MD, Ashraful Islam Mollab. Preparation of Chitosan from Shrimp Shell and Investigation of its Properties. Int J Basic Appl Sci 2011;11:16-130.

21. Black CA. Methods of Soil Analysis. Part I physical and mineralogical properties. Madison (WI) USA: American Society of Agronomy; 1965.

22. Li Q, Dunn ET, Grandmaison EW, Goosen MFA. Applications and properties of chitosan. J Bioact Compat Polym 1992;7:370-97.

23. Park JH, Saravanakumar G, Kim KK. Targeted delivery of low molecular drugs using chitosan and its derivatives. Adv Drug Deliv Rev 2010;62:28-41.

24. New Wall CA, Anderson LA, Phillipsan JD. Herbal medicines-A guide for healthcare professionals. 3rd ed. London: The Pharmaceutical Press; 1996.

25. Tusji A. Specific Mechanisms for Transporting Drugs into Brain. In: Begley DJ, Bradbury MW, Kreuter J, editors. The blood-brain barrier and drug delivery to the CNS. New York: Marcel Dekker Inc; 2000. p. 121-44.

26. Schinkel AH. P-Glycoprotein, a gatekeeper in the blood-brain barrier. Adv Drug Deliv Rev 1999;36:179-94.

27. Taylor EM. The impact of efflux transporters in the brain on the development of drugs for CNS disorder. Clin Pharm Kinet 2002;41:81-92.

28. Issa MM, Koping-Hoggard M, Artursson P. Chitosan and the mucosal delivery of biotechnology drugs. Drug Discov Today Technol 2005;2:1-6.

29. Ranaldi G, Marigliano I, Vespignani I. The effect of chitosan and other polycations on tight junction permeability in the human intestinal Caco-2 cell line. J Nutr Biochem 2002;13:157-67.

30. Tolimate A, Desbrieres J, Rhazi M, Alagui A, Vincendon M, Vottero P. On the influence of deacetylation process on the physicochemical characteristics of chitosan from squid chitin. Polymer 2000;41:2463-9.

31. de Vasconcelos CL, Bezerril PM, Dantas TNC, Pereira MR, Fonseca JLC. Adsorption of bovine serum albumin on template polymerized chitosan/poly (methacrylic acid) complexes. Langmuir 2007;23:7687-94.

32. Xu X, Ding Y, Qian Z, Wang F, Wen B, Zhou H, et al. Degradation of poly (ethylene terephthalate)/clay nanocomposites during melt extrusion: Effect of clay catalysis and chain extension. Polym Degrad Stab 2009;94:113-23.

33. Mano JF, Koniarova D, Reis RL. Materials in Medicine. J Mater Sci 2003;14:127-35.

34. Juliano RL, Ling VA. Surface glycoprotein modulating drug permeability in Chinese hamster ovary cell mutants. Biochem Biophys Acta 1976;455:152-62.

35. Alicia JS, Ching AP. Ring-Opening Polymerization of $\varepsilon$-Caprolactone Initiated by Ganciclovir (GCV) for the Preparation of GCV-Tagged Polymeric Micelles. Molecules 2015;20:2857-67.

36. Anita Margret A, Aishwarya S, Violet Dhayabaran V. Steering up brain drug targeting: an in silico approach to combat the drug 
efflux permeability glycoprotein (P-gp) with antidepressant drug venlafaxin and phytocompounds of Clitoria ternate. Int $\mathrm{J}$ Univers Pharm Life Sci 2013;2:186-9.

37. Wang J, Hou T. Recent Advances on Aqueous Solubility Prediction. Comb Chem High Throughput Screen 2011;14:1-11.

38. James L, Mc Donough. Uniting Cheminformatics and
Chemical Theory to Predict the Intrinsic Aqueous Solubility of Crystalline Drug like Molecules. J Chem Inf Model 2013;54:844-56.

39. Kujawski J, Popielarska H, Myka A, Drabinska B, Bernard MK. The $\log \mathrm{P}$ Parameter as a Molecular Descriptor in the Computer-aided Drug Design - an Overview. Comput Methods Sci Technol 2012;18:81-8. 